\title{
Feedback effects of dynamic hedging strategies in the presence of transaction costs
}

\author{
Elettra Agliardi*, Rainer Andergassen** \\ Department of Economics, University of Bologna \\ Piazza Scaravilli 2, 40126 Bologna \\ *agliardi@economia.unibo.it \\ **anderga@economia.unibo.it
}

\begin{abstract}
We study the destabilising effect of dynamic hedging strategies on the price of the underlying in the presence of sunk costs of transaction. Once sunk costs of transaction are taken into account, continuous portfolio rehedging is no longer an optimal strategy. Using a non-optimising (local in time) strategy for portfolio rebalancing, explicit dynamics for the price of the underlying are derived, focusing in particular on the excess volatility and feedback effects of these portfolio insurance strategies. Further, we show how these latter depend on the heterogeneity of the insured payoffs. Finally, conditions are derived under which it may still be reasonable, from a practical viewpoint, to implement Black - Scholes strategies.

J.E.L. Classification numbers: G10, G11, G12.

Keywords: dynamic hedging, volatility, Black-Scholes model, transaction costs.
\end{abstract}




\section{Introduction}

Standard option pricing literature relies on the hypothesis that the dynamics of the underlying asset are independent of the hedging strategy. Dynamic delta hedging strategies require to sell the underlying asset if its price decreases, while they require to buy if its price increases. The hypothesis of independency between strategies and price dynamics of the underlying asset corresponds to the assumption that the market for the underlying asset is perfectly liquid.

Positive feedback effects from dynamic delta hedging strategies have been studied recently assuming that the asset market for the underlying asset is only finitely liquid (see, for example, Frey and Stremme (1997), Schoenbucher and Wilmott (2000) and their references). It has been shown that in this case portfolio insurance activity has a destabilising effect on the dynamics of the price of the underlying asset. In particular, these strategies increase the volatility of the price of the underlying asset. The above-mentioned papers assume that program traders can buy and sell assets without incurring transaction costs. But, as a matter of fact, transaction costs are non-negligible in asset markets.

If we introduce transaction costs, then it is no longer optimal to adjust the portfolio continuously. There are two main approaches in the literature taking the effects of transaction costs into account: the first considers discrete adjustments of the portfolio, where the time step of portfolio rebalancing is exogenously given, while the second considers traders as continuously monitoring the price of the underlying asset, although adjusting their portfolio only if the gain from adjustment is greater than the cost of adjustment. This latter approach can be subdivided into two further approaches: the first is called local in time, while the second is called global in time. The former is a non-optimising approach, while the latter is an optimising one (see, for example, Wilmott (2000) for a review).

In this paper we will follow a local in time approach and assume that transaction costs are sunk costs ${ }^{1}$.

Suppose that an agent's portfolio is given by a long position on a call option and a short position on some units of the underlying asset, such that a riskless portfolio is obtained. If we denote by $V(S, t)$ the option value, where $S$ is the value of the underlying asset, then the perfect Black-Scholes hedge is given by

$$
\Delta=\frac{\partial V}{\partial S}>0
$$

Consider now a change in the price of the underlying asset. Given this change, in the absence of transaction costs, it would be optimal to rehedge the position in order to maintain a riskless portfolio. But once we assume that there are sunk costs of adjustment, it is no longer optimal to rehedge immediately the portfolio as the price of the asset changes. Thus, we have to define a new strategy for portfolio rehedging.

\footnotetext{
${ }^{1}$ The model could be extended trivially in order to consider also proportional transaction costs.
} 
Following Whalley and Wilmott (1993) and Henrotte (1993) we define a confidence level for the deviation of the risky asset position from the perfect hedge. Suppose an agent $i$ holds $-G^{i}$ units of the underlying risky asset. The risk of the imperfectly hedged position, measured by the variance over a timestep $\delta t$, is given by $\sigma^{2} S^{2}\left(G^{i}-\frac{\partial V^{i}}{\partial S}\right)^{2} \delta t$, to leading order. If such position is perfectly hedged, i.e., $G^{i}=\frac{\partial V^{i}}{\partial S}$, then the portfolio risk is eliminated completely, otherwise the position is still risky.

We have now to determine the rehedging strategy. Define $\eta^{i}(S, \tau, K)=$ $\frac{\partial V^{i}}{\partial S}-G^{i}$ as the hedge-unbalance level of agent $i$, where $\tau=T-t$ denotes time to maturity and $K$ is the strike price. Whalley and Wilmott (1993) and Henrotte (1993) define a tolerance level $\tilde{H}_{0}^{i}$ such that

$$
\sigma S\left|G^{i}-\frac{\partial V^{i}}{\partial S}\right| \leqslant \tilde{H}_{0}^{i}
$$

where inaction is optimal as long as (2) is satisfied, while the position should be rebalanced once (2) is violated. The parameter $\tilde{H}_{0}^{i}$ gives a measure of the maximum expected risk in the portfolio.

In what follows, we will assume that $\tilde{H}_{0}^{i}$ is partly deterministic and partly stochastic: the former captures the influence of transaction costs, while the latter captures stochastic contingencies. Given these assumptions, we can define an adjustment hazard function for each program trader. Following Caballero and Engel $(1993,1999)$ we will study the aggregate dynamics of the adjustment hazard rates and the resulting price dynamics of the risky asset.

The paper is organised as follows. In Section 2 the adjustment hazard function is formally introduced and the model is presented. Section 3 contains the main results. Finally, Section 4 concludes.

\section{The basic model}

Suppose that there are two types of traders operating in a market, where there is a risky asset and a riskless one (a pure discount bond): program traders and reference traders. Program traders use a dynamic hedging strategy, while reference traders are small price takers, which include market makers and market timers, providing liquidity for market transactions. We assume that there is a continuum of reference traders, such that the effects of transaction costs on the aggregate demand function of reference traders are negligible. In other words, we can assume a smooth aggregate demand function with respect to the price of the risky asset. Furthermore, we suppose that reference traders have perfect information about the fundamentals of the risky asset. The aggregate demand function of reference traders is denoted by $D\left(t, F_{t}, S_{t}\right)$, where $S$ is the proposed price of the risky asset, while $F$ can be interpreted in different ways. For example, Frey and Stremme (1997) assume $F$ to be the aggregate income of reference traders, while Platen and Schweizer (1994) assume $F$ to be an unspecified liquidity demand, and others assume $F$ to be the fundamental value 
of the firm. We follow this latter approach, and make the following assumptions about the demand of reference traders:

\section{Assumption 1.}

a) $D\left(t, F_{t}, S_{t}\right)$ is a smooth function

b) $\frac{\partial D}{\partial S} \leqslant-d<0$ and $\frac{\partial D}{\partial F}>0$

Assumption 1.b). indicates that as the price of the asset increases, demand decreases, while as the fundamental value of the asset increases, demand increases as well.

Let us normalize the total supply of the risky asset to one. Thus, in the absence of program traders, equilibrium is guaranteed by the following market clearing condition

$$
D\left(t, F_{t}, S_{t}^{*}\right)=1
$$

From (3) it follows that the equilibrium price of the asset is $S_{t}^{*}=\varphi\left(t, F_{t}\right)$. Thus, the price of the asset follows its fundamental.value. We will call $S_{t}^{*}$ the normal price of the asset.

We are looking for a diffusion process for the asset price of the form

$$
d S_{t}=\mu_{S}\left(S_{t}, t\right) d t+\sigma_{S}\left(S_{t}, t\right) d W_{t}
$$

where $W_{t}$ denotes a Wiener process. Once we have specified the demand function of the reference traders and the stochastic process for the fundamental value we can determine the dynamics of the asset price $S_{t}^{*}$.

We will assume that the dynamics of the fundamental value of the risky asset follow a diffusion process of the form

$$
d F_{t}=\mu_{F}\left(F_{t}, t\right) d t+\sigma_{F}\left(F_{t}, t\right) d W_{t} .
$$

Using the equilibrium condition (3), the dynamics of the fundamental value (5) and the fact that we are looking for a diffusion process for the risky asset of the type (4), we have that, in equilibrium, the following condition has to be satisfied

$$
\begin{gathered}
0=\left(D_{S} \sigma_{S}\left(S_{t}^{*}, t\right)+D_{F} \sigma_{F}\left(F_{t}, t\right)\right) d W_{t}+ \\
+\left(D_{S} \mu_{S}\left(S_{t}^{*}, t\right)+D_{F} \mu_{F}\left(F_{t}, t\right)+\right. \\
+\frac{1}{2} D_{S S}\left(\sigma_{S}\left(S_{t}^{*}, t\right)\right)^{2}+\frac{1}{2} D_{F F}\left(\sigma_{F}\left(F_{t}, t\right)\right)^{2}+ \\
\left.+D_{S F} \sigma_{S}\left(S_{t}^{*}, t\right) \sigma_{F}\left(F_{t}, t\right)+D_{t}\right) d t
\end{gathered}
$$

In order to satisfy condition (6) we need the stochastic as well as the deterministic term in equation (6) equal to zero; therefore, we obtain the following moments for the risky asset price dynamics:

$$
\sigma_{S}\left(S_{t}^{*}, t\right)=-\sigma_{F}\left(F_{t}, t\right) \frac{D_{F}}{D_{S}}
$$




$$
\begin{aligned}
\mu_{S}\left(S_{t}^{*}, t\right)= & -\frac{1}{D_{S}}\left[D_{t}+D_{F} \mu_{F}\left(F_{t}, t\right)+\frac{1}{2} D_{S S}\left(\sigma_{F}\left(F_{t}, t\right) \frac{D_{F}}{D_{S}}\right)^{2}+\right. \\
& \left.+\frac{1}{2} D_{F F}\left(\sigma_{F}\left(F_{t}, t\right)\right)^{2}-D_{S F}\left(\sigma_{F}\left(F_{t}, t\right)\right)^{2} \frac{D_{F}}{D_{S}}\right]
\end{aligned}
$$

Thus, the price dynamics of the risky asset $S_{t}^{*}$ follow a diffusion process (4), where $\sigma_{S}\left(S_{t}^{*}, t\right)$ and $\mu_{S}\left(S_{t}^{*}, t\right)$ are given by expressions $(7)$.

In the next Section we are going to study the aggregate demand of program traders. Then, we plug the aggregate demand of program traders into expression (3) and study its implications for the price dynamics of the risky asset.

\subsection{Aggregate demand of program traders}

We argued in Section 1 that in the case of no transaction costs, continuous adjustment of the portfolio is optimal. But once we introduce transaction costs, and in particular sunk costs of adjustment, continuous adjustment of the portfolio is no longer optimal.

Let us define a confidence level $\tilde{H}_{0}$ as in expression (2), and suppose it is a function $\tilde{H}_{0}\left(c, H_{0}\right)$, where $c \geqslant 0$ is the deterministic component, while $H_{0}$ is the stochastic component. The deterministic component $c$ captures the influence of the size of the transaction costs on the confidence level. An increase in the transaction costs increases $c$. In particular, $c$ may depend on the size of the bidask spread. The stochastic component $H_{0}$ captures the influence of stochastic contingencies on the confidence level.

We define a probability of adjusting the portfolio in the following way

$$
\operatorname{Pr}\left(\tilde{H}_{0}\left(c, H_{0}\right) \leqslant\left|\eta_{t+d t}\right||| \eta_{t} \mid<\tilde{H}_{0}\left(c, H_{0}\right)\right)=h(\eta, c) d t
$$

where $\eta=\eta(S, \sigma, \tau, K)=\Delta(S, \sigma, \tau, K)-G, \Delta(S, \sigma, \tau, K)=\frac{\partial V(S, \sigma, \tau, K)}{\partial S}, \sigma$ is the input volatility, i.e. the volatility used for the computation of the hedging strategy (Frey and Stremme, 1997), $\tau$ is the time to maturity, while $K$ is the strike price.

We assume that the distribution of $H_{0}$ is the same for each program trader, and thus, the probability of portfolio rehedging depends just on the hedging unbalance level $\eta$ and on the level of the deterministic component $c$. We make the following assumptions about the adjustment hazard function $h(\eta, c)$ :

\section{Assumption2.}

a) $h(\cdot)$ is a smooth function

b) $h_{\eta}(\eta, c)>0$, and $h_{c}(\eta, c)<0$

c) $\lim _{c \rightarrow 0} h(\eta, c)=1$ and $\lim _{c \rightarrow \infty} h(\eta, c)=0$. 
Assumption 2.b) states that the probability of adjustment increases as the hedging unbalance level increases and/or as the size of the transaction costs decreases. Assumption 2.c) implies that, if the size of the transaction costs is vanishing small, then the probability of portfolio adjustment converges towards one, that is, we have continuous adjustment or dynamic delta hedging, while, if the transaction costs are infinitely large, then the probability of portfolio adjustment becomes vanishing small.

The hedging unbalance level $\eta$ has a common element for each program trader, which is the price of the underlying $S$, while there are also idiosyncratic components such as the strike price $K$ and time to maturity $\tau$. Thus, as the price of the underlying asset changes, the hedging unbalance level for each program trader $i, \eta^{i}$, changes as well, while the way it changes depends on the distribution of strike prices and of the time to maturity.

By aggregating program traders over their hedging unbalance levels we have that the average demand of the risky asset over a time interval $d t$ becomes

$$
g(S, \sigma, c, t)=\int_{\Re} \eta h(\eta, c) f(\eta) d \eta
$$

where $f(\eta)$ is the distribution of the hedging unbalance level over the program traders. This latter distribution is not time-invariant since, as the time goes on $\tau$ changes, and thus, according to the distribution of $\tau$, the distribution of $\eta$ changes. We are going to assume a continuous and random influx and outflux of program traders from the asset market, and heterogeneity in the distribution of strikes. Thus, we have that the average demand over a small time period $d t$ of program trader is given by

$$
\Psi(S, \sigma, c)=\int_{\Re_{+}^{2} \times R} \eta(S, \sigma, \tau, K) h(\eta(S, \sigma, c, \tau, K)) f(\eta) v(d K \oplus d \tau \oplus d \eta)
$$

where $v$ has a smooth density function with respect to a Lebesgue-measure. Thus, expression (8) represents the demand for the underlying asset of the program traders over a small time period $d t$. We are interested to see how this demand changes as the price of the underlying changes.

Consider the change in the price of the risky asset of size $d S$. Using expression (8) we have:

$$
\begin{gathered}
\Psi(S+d S, \sigma, c)=\int_{\Re_{+}^{2} \times R}[\Delta(S+d S, \sigma, \tau, K)-\Delta(S, \sigma, \tau, K)+\eta] \times \\
\times h(\Delta(S+d S, \sigma, \tau, K)-\Delta(S, \sigma, \tau, K)+\eta, c) \times \\
\times f(\eta) v(d K \oplus d \tau \oplus d \eta)
\end{gathered}
$$

Taking Taylor expansion of $\Delta(S+d S,$.$) and h(\Delta(S+d S,)-.\Delta(S,)+.\eta)$ around $S$ and $\eta$ respectively, we have

$$
\Delta(S+d S, .)-\Delta(S, .)=\Delta_{S} d S+\frac{1}{2} \Delta_{S S}(d S)^{2}
$$


and

$$
\begin{gathered}
h(\Delta(S+d S, .)-\Delta(S, .)+\eta, c)=h(\eta, c)+h_{\eta}(\eta, c)\left(\Delta_{S} d S+\frac{1}{2} \Delta_{S S}(d S)^{2}\right)+ \\
+\frac{1}{2} h_{\eta \eta}(\eta, c)\left(\Delta_{S} d S+\frac{1}{2} \Delta_{S S}(d S)^{2}\right)^{2}
\end{gathered}
$$

Now we can calculate $d \Psi(S, \sigma, c)=\Psi(S+d S, \sigma, c)-\Psi(S, \sigma, c)$ as follows

$$
\begin{gathered}
d \Psi(S, \sigma, c)=\int_{\Re_{+}^{2} \times R}\left\{[ \Delta _ { S } d S + \frac { 1 } { 2 } \Delta _ { S S } ( d S ) ^ { 2 } ] \left[h(\eta, c)+h_{\eta}(\eta, c)\left(\Delta_{S} d S+\right.\right.\right. \\
\left.\left.+\frac{1}{2} \Delta_{S S}(d S)^{2}\right)+\frac{1}{2} h_{\eta \eta}(\eta, c)\left(\Delta_{S} d S+\frac{1}{2} \Delta_{S S}(d S)^{2}\right)^{2}\right]+ \\
+\eta h_{\eta}(\eta, c)\left(\Delta_{S} d S+\frac{1}{2} \Delta_{S S}(d S)^{2}\right)+ \\
\left.+\frac{1}{2} \eta h_{\eta \eta}(\eta, c)\left(\Delta_{S} d S+\frac{1}{2} \Delta_{S S}(d S)^{2}\right)^{2}\right\} f(\eta) v(d K \oplus d \tau \oplus d \eta)
\end{gathered}
$$

Since $(d S)^{\theta}=0$ for $\theta>2$ we obtain, after rearranging terms

$$
\begin{gathered}
d \Psi(S, \sigma, c)=d S \int_{\Re_{+}^{2} \times R}\left[h(\eta, c)+\eta h_{\eta}(\eta, c)\right] \Delta_{S} f(\eta) v(d K \oplus d \tau \oplus d \eta)+ \\
+\frac{1}{2}(d S)^{2} \int_{\Re_{+}^{2} \times R}\left\{\left[h(\eta, c)+\eta h_{\eta}(\eta, c)\right] \Delta_{S S}+\right. \\
\left.+\left[2 h_{\eta}(\eta, c)+\eta h_{\eta \eta}(\eta, c)\right]\left(\Delta_{S}\right)^{2}\right\} f(\eta) v(d K \oplus d \tau \oplus d \eta)
\end{gathered}
$$

This latter expression can be rewritten as follows

$$
d \Psi(S, \sigma, c)=H_{1}(S, \sigma, c) d S+\frac{1}{2} H_{2}(S, \sigma)(d S)^{2}
$$

where

$$
\begin{gathered}
H_{1}(S, \sigma, c)=\int_{\Re_{+}^{2} \times R} \tilde{h}(\eta, c) \Delta_{S}(S, \sigma, K, \tau) f(\eta) v(d K \oplus d \tau \oplus d \eta) \\
H_{2}(S, \sigma, c)=\int_{\Re_{+}^{2} \times R}\left\{\tilde{h}(\eta, c) \Delta_{S S}+\tilde{h}_{\eta}(\eta, c)\left(\Delta_{S}\right)^{2}\right\} f(\eta) v(d K \oplus d \tau \oplus d \eta)
\end{gathered}
$$

and where $\tilde{h}(\eta, c)=\frac{\partial}{\partial \eta} \eta h(\eta, c)=h(\eta, c)+\eta h_{\eta}(\eta, c)$ and $\tilde{h}_{\eta}=\frac{\partial}{\partial \eta} \tilde{h}(\eta, c)=$ $2 h_{\eta}(\eta, c)+\eta h_{\eta \eta}(\eta, c)$.

Let us introduce the following assumption:

Assumption 3. There exist functions $v_{1}$ and $v_{2}$ such that $v(d K \oplus d \tau \oplus d \eta)=$ $v_{1}(d \eta) v_{2}(k, \tau) d K d \tau$ where $v_{1}$ and $v_{2}$ have a smooth density function with respect to a Lebesgue measure; $v_{2}$ has a compact support in $R_{+} \times[0, \infty)$. 
With Assumption 3, we can rewrite expression (10) as follows

$$
H_{1}(S, \sigma, c)=\tilde{H}(c) \tilde{\Gamma}(S, \sigma)
$$

where

$$
\begin{gathered}
\tilde{H}(c)=\int_{\Re} \tilde{h}(\eta, c) f(\eta) v_{1}(d \eta) \\
\tilde{\Gamma}(S, \sigma)=\int_{\Re_{+}^{2}} \Delta_{S}(S, \sigma, K, \tau) v_{2}(k, \tau) d K d \tau
\end{gathered}
$$

$\tilde{H}(c)$ indicates the stationary average size of the adjustment, given a change in the hedge unbalance level. $\Delta_{S}(S, \sigma, K, \tau)$ is known in the option pricing literature as the parameter gamma, and it indicates, in the absence of transaction costs, how often a position must be rehedged in order to maintain a delta-neutral position. Thus, $\tilde{\Gamma}(S, \sigma)$ is the stationary average value of the gamma, which indicates, in the absence of transaction costs, how often in the stationary state, a position must be adjusted on average in order to keep delta-neutral positions.

$H_{1}(S, \sigma, c)$ indicates the stationary average adjustment, given a change in the price of the risky asset. $H_{1}(S, \sigma, c)$ depends: a) on the average size of adjustment, and thus on the properties of the adjustment hazard function and on the stationary state distribution of unbalance levels, and b) on the frequency of adjustment, which depends on the stationary average sensibility of the delta with respect to the price of the underlying. Notice that Assumption 2 implies that $\frac{\partial}{\partial c} H_{1}(S, \sigma, c)<0$ and $\lim _{c \rightarrow 0} H_{1}(S, \sigma, c)=\tilde{\Gamma}(S, \sigma)$, while $\lim _{c \rightarrow \infty} H_{1}(S, \sigma, c) \stackrel{0}{=}$. In other words, $H_{1}(S, \sigma, c)$ is a decreasing function of $c$.

Furthermore, if the size of the transaction costs is vanishing small, then $H_{1}(S, \sigma, c)$ converges towards the stationary average value of the gamma and so we are back to the case of dynamic hedging strategies, while if the transaction costs are very large, then no portfolio adjustment occurs at all. Finally, by Assumption 2 we have $H_{1}(S, \sigma, c) \geqslant 0$.

\section{Positive feedback effects from hedging}

As we pointed out before, reference traders have perfect information about the fundamental value of the risky asset. Thus, a reduction in the fundamental value leads to a decrease in the price of the risky asset. Given this decrease, some program traders will sell the risky assets in order to adjust their portfolio. This latter leads to a further price reduction of the risky asset, which now will be lower than its normal level, i.e. $S_{t}<S_{t}^{*}$. Thus, the action of program traders leads to potential gains for liquidity providers, such as market makers and market timers (see Grossman , 1988). These latter could buy the assets since their actual price is now lower than their normal price. In this way, liquidity 
providers have a stabilising function. Such ability to exploit gains from excess volatility of price dynamics, depends on some parameters, for example, the cost of capital, the transaction costs $(c)$ and also the information about how many agents are using a dynamic hedging strategy. If these liquidity providers commit insufficient capital, then their stabilising function will be reduced.

Let us indicate by $\rho(c, \Im) \in[0,1]$ the weight of the program traders, that is, a measure of market liquidity. $\rho(c, \Im)$ is a function of $c$, the transaction costs, and $\Im \in \Re_{+}$, which captures the effects of other variables on market liquidity, such as lack of information on hedging activity and cost of capital. We take $\Im$ and $c$ as exogenous variables. We make the following assumptions about the behaviour of $\rho(c, \Im)$

\section{Assumption 4.}

a) $\frac{\partial \rho}{\partial c}>0, \frac{\partial \rho}{\partial \Im}>0$

b) $\lim _{c \rightarrow \infty} \rho(c, \Im)=1$

c) $\lim _{c \rightarrow 0, \Im \rightarrow 0} \rho(c, \Im)=0$

d) $\lim _{c \rightarrow 0} \rho(c, \Im) \geqslant 0$ and $\lim _{c \rightarrow 0} \rho(c, \Im)>0$ as long as $\Im>0$.

Assumption 4.a) implies that an increase in the transaction costs and in the exogenous parameter $\Im$ reduces the liquidity of the market; 4..b) implies that as the transaction costs diverge towards infinity, the market is completely illiquid; 4.c) implies that the market is perfectly liquid if the supply of capital is perfectly elastic, information is perfect and if there are no transaction costs; 4.d) implies that if transaction costs are vanishing small, then the market will still be illiquid, where the size of illiquidity depends on the size of the exogenous variable $\Im$.

Let us plug $\rho(c, \Im)$ into the demand of reference traders. Using the market clearing condition, we have that the equilibrium price has to satisfy the following condition

$$
D\left(t, F_{t}, S_{t}\right)+\rho(c, \Im) \Psi\left(S_{t}, \sigma, c\right)=1
$$

According to Assumption 4, for $(c, \Im) \rightarrow 0$ the action of the program traders has a negligible effect on the price dynamics of the risky asset. Thus, each deviation of prices from their normal level will be eliminated through the action of market timers. On the other side, if the supply of capital is not perfectly elastic, and market timers do not have perfect information, then market timers cannot completely eliminate the effect of the action of the program traders (see Grossman , 1988). Thus, as long as $\rho(c, \Im)>0$ the market is only finitely liquid. Notice that an increase in the transaction costs increases the weight of the portfolio insurance in the aggregate demand, i.e. the LHS of expression (14).

Now we can prove the main result. 
Proposition 1 The diffusion process governing the dynamics of the asset price is of the form (4) with parameters:

$$
\begin{gathered}
\sigma_{S}\left(S_{t}, t ; \sigma, c\right)=-\frac{D_{F}}{D_{S}+\rho(c, \Im) \tilde{H}(c) \tilde{\Gamma}\left(S_{t}, \sigma\right)} \sigma_{F}\left(F_{t}, t\right) \\
\mu_{S}\left(S_{t}, t ; \sigma, c\right)=-\frac{1}{D_{S}+\rho(c, \Im) \tilde{H}(c) \tilde{\Gamma}\left(S_{t}, \sigma\right)}\left[D_{t}+D_{F} \mu_{F}\left(F_{t}, t\right)+\right. \\
-D_{S F} \frac{D_{F}}{D_{S}+\rho(c, \Im) \tilde{H}(c) \tilde{\Gamma}\left(S_{t}, \sigma\right)}\left(\sigma_{F}\left(F_{t}, t\right)\right)^{2}+ \\
+\frac{1}{2}\left(D_{S S}+\rho H_{2}\left(S_{t}, \sigma, c\right)\right)\left(\frac{D_{F}}{D_{S}+\rho(c, \Im) \tilde{H}(c) \tilde{\Gamma}\left(S_{t}, \sigma\right)}\right)^{2} \times \\
\left.\times\left(\sigma_{F}\left(F_{t}, t\right)\right)^{2}+D_{F} \mu_{F}\left(F_{t}, t\right)\right]
\end{gathered}
$$

Proof. Taking total differential of (14) and using (9) we have that

$$
\begin{gathered}
0=D_{t}+D_{S} d S+D_{F} d F+\frac{1}{2} D_{S S}(d S)^{2}+D_{S F} d S d F+\frac{1}{2} D_{F F}(d F)^{2}+ \\
\rho(c, \Im) H_{1}\left(S_{t}, \sigma, c\right) d S+\frac{1}{2} \rho(c, \Im) H_{2}\left(S_{t}, \sigma, c\right)(d S)^{2}
\end{gathered}
$$

Using the stochastic process (5) we can rewrite this condition as follows

$$
\begin{gathered}
0=\left[D_{t}+D_{S} \mu_{S}\left(S_{t}, t\right)+D_{F} \mu_{F}\left(F_{t}, t\right)+\frac{1}{2} D_{S S}\left(\sigma_{S}\left(S_{t}, t\right)\right)^{2}+\right. \\
+D_{S F} \sigma_{S}\left(S_{t}, t\right) \sigma_{F}\left(F_{t}, t\right)+\frac{1}{2} D_{F F}\left(\sigma_{F}\left(F_{t}, t\right)\right)^{2}+ \\
\left.+\rho(c, \Im) H_{1}\left(S_{t}, \sigma, c\right) \mu_{S}\left(S_{t}, t\right)+\frac{1}{2} \rho H_{2}\left(S_{t}, \sigma, c\right)\left(\sigma_{S}\left(S_{t}, t\right)\right)^{2}\right] d t+ \\
+\left[D_{F} \sigma_{F}\left(F_{t}, t\right)+D_{S} \sigma_{S}\left(S_{t}, t\right)+\rho(c, \Im) H_{1}\left(S_{t}, \sigma, c\right) \sigma_{S}\left(S_{t}, t\right)\right] d W_{t}
\end{gathered}
$$

Since (16) has to be true, we need the deterministic as well as the stochastic term in (16) to be equal to zero. Thus, we obtain the values in expression (15) for $\sigma_{S}\left(S_{t}, t\right)$ and $\mu_{S}\left(S_{t}, t\right)$. Obviously, we need $\sigma_{S}\left(S_{t}, t\right) \geqslant 0$, and this is true for appropriate values of $d$ in Assumption 1. Thus the price of the underlying follows a non-linear diffusion process (4).

From expression (15) in Proposition 1 we observe that the larger the average gamma, the larger the volatility of the asset price of the underlying asset. Since gamma indicates how often a position must be rehedged on average in order to maintain a delta-neutral position, the higher is its average value, the more frequently an adjustment occurs. At the same time, $\tilde{H}(c)$ indicates the average size of the adjustment, given a change in the hedge unbalance level. This latter depends on the properties of the adjustment hazard function and on the size of the deterministic component of the confidence level $c$. This latter component depends directly on the size of the transaction costs. Thus, the larger the transaction costs, the lower $\tilde{H}(c)$. On the other side, the larger the transaction costs, the less liquid the market, and therefore the weight of the demand of the portfolio insurance in the aggregate demand of the risky asset increases. Thus, the effect of a change in the transaction costs on the volatility of the underlying asset is a priori ambiguous. Given Assumptions 2 and 4 we have that if the 
transaction costs are infinitely high, then $\lim _{c \rightarrow \infty} \rho(c, \Im) \tilde{H}(c)=0$ and so on average no portfolio adjustment occurs and there will be no feedback effect, i.e. $S_{t}=S_{t}^{*}$. On the other side, if transaction costs are vanishing small, then, since $\lim _{c \rightarrow 0} \tilde{H}(c)=1$, we are back to dynamic hedging strategies where the liquidity of the market $\rho(0, \Im)$ depends just on the size of $\Im$, and thus we are back to a situation like the one studied by Frey and Stremme (1997).

The volatility of the underlying asset will be larger, the larger will be the frequency of adjustment and/or the larger will be the size of adjustment. The volatility depends also on $\rho(c, \Im)$. In particular, the more liquid the market, the lower the influence of the hedging activity of the program traders on the price dynamics of the underlying asset.

Notice that as long as $\rho(c, \Im)>0$ and $c<\infty$ the price dynamics of the risky asset $S_{t}$ are different from their normal counterpart $S_{t}^{*}$. In particular, comparing (15) with (7) we observe that there exists an excess volatility due to the hedging activity of program trader. The size of the excess volatility depends on the liquidity of the market and on the aggregate characteristics of program traders, i.e. $\tilde{H}(c) \tilde{\Gamma}\left(S_{t}, \sigma\right)$. But since expression (15) for the volatility still depends on the input volatility $\sigma$, consistency requires that input volatility be equal to the actual observed volatility. In other words, we have to solve a fixed point problem.

In solving the fixed point problem, we will make use of the following Lemma.

Lemma (i) $\tilde{\Gamma}$ is a bounded function of $\sigma$; (ii) for $\sigma \geqslant \sigma_{0}$, with $0<\sigma_{0}<\infty$, $\frac{\partial}{\partial \sigma} \tilde{\Gamma}$ is a bounded function of $\sigma$.

Proof. (i) The following equalities hold, because of the definition of $\Delta$ and Assumption 3:

$$
\begin{gathered}
\tilde{\Gamma}=\iint \frac{\partial \Delta}{\partial S} v_{2}(K, \tau) d K d \tau=-\iint \frac{K}{S} \frac{\partial \Delta}{\partial K} v_{2}(K, \tau) d K d \tau \\
\iint \Delta \frac{\partial}{\partial K}\left(\frac{K}{S} v_{2}\right) d K d \tau
\end{gathered}
$$

since $v_{2}$ has a compact support. Since $0 \leqslant \Delta \leqslant 1$, we get:

$$
|\tilde{\Gamma}| \leqslant \iint\left|\frac{\partial}{\partial K}\left(\frac{K}{S} v_{2}\right)\right| d K d \tau
$$

that is, $\tilde{\Gamma}$ is a bounded function of $\sigma$.

(ii) Recall that $\frac{\partial \Delta}{\partial S}=\frac{N^{\prime}\left(d_{1}\right)}{\sigma S \sqrt{\tau}}$, where $d_{1}=\frac{\ln \left(\frac{S}{K}\right)+\left(r+\frac{1}{2} \sigma^{2}\right) \tau}{\sigma \sqrt{\tau}}$. Then, $\frac{\partial}{\partial \sigma}\left(\frac{N^{\prime}\left(d_{1}\right)}{\sigma S \sqrt{\tau}}\right) \leqslant$ $N^{\prime \prime}\left(d_{1}\right) \frac{1}{\sigma S \sqrt{\tau}}-\frac{N^{\prime}\left(d_{1}\right)}{\sigma^{2} S \sqrt{\tau}} \leqslant \frac{1}{\sigma_{0} S \sqrt{\tau}}+\frac{1}{\sigma_{0}^{2} S \sqrt{\tau}}$, that is, $\frac{\partial}{\partial \sigma} \tilde{\Gamma}$ is a bounded function of $\sigma$ for $\sigma>\sigma_{0}$.

We are now able to state and proof the following Proposition. 
Proposition 2 Under Assumptions 1-4, there exists a solution of the fixed point problem $\sigma_{S}\left(S_{t}, t ; \sigma, c\right)=-\frac{D_{F}}{D_{S}+\rho(c, \Im) \tilde{H}(c) \tilde{\Gamma}\left(S_{t}, \sigma\right)} \sigma_{F}\left(F_{t}, t\right)$ provided that $\rho$ is sufficiently small.

Proof. Let us put $M(\sigma)=\sigma_{S}(S, t ; \sigma, c)=-\frac{D_{F} \sigma_{F}\left(F_{t}, t\right)}{D_{S}+\rho(c, \Im) \tilde{H}(c) \tilde{\Gamma}\left(S_{t}, \sigma\right)}$. We have to show that $\left|\frac{\partial M(\sigma)}{\partial \sigma}\right| \leqslant M<1$, with $0 \leqslant M \leqslant 1$, in order to apply the contraction mapping theorem.

We have that:

$$
\left|\frac{\partial M(\sigma)}{\partial \sigma}\right|=\left|\frac{D_{F} \sigma_{F} \rho\left(\frac{\partial \tilde{H}}{\partial \sigma} \tilde{\Gamma}+\tilde{H} \frac{\partial \tilde{\Gamma}}{\partial \sigma}\right)}{\left(D_{S}+\rho \tilde{H} \tilde{\Gamma}\right)^{2}}\right|
$$

Let us first consider the denominator. If $|\rho|<\varepsilon$ we get, for some $\tilde{e}$ :

$$
\left|D_{S}+\rho \tilde{H} \tilde{\Gamma}\right| \geqslant\left|D_{S}\right|-|\rho||\tilde{H} \tilde{\Gamma}| \geqslant d-\varepsilon \tilde{e} \geqslant \frac{d}{2}
$$

provided that $\varepsilon \leqslant \frac{d}{2 \tilde{e}}$. (17) holds because of Assumption 1, the Lemma, and in view of the fact that $\tilde{H}$ is a bounded function of $\sigma$, since $0 \leqslant \Delta \leqslant 1$, and $h(\eta, c), f(\eta), v_{1}(d \eta)$ are bounded functions of $\sigma$.

Let us consider the numerator. We get, with suitable constants $\widetilde{\widetilde{e}}, J$ :

$$
\begin{gathered}
\left|-D_{F} \sigma_{F} \rho\left(\frac{\partial \tilde{H}}{\partial \sigma} \tilde{\Gamma}+\tilde{H} \frac{\partial \tilde{\Gamma}}{\partial \sigma}\right)\right|= \\
=\left|D_{F} \sigma_{F}\right||\rho|\left|\frac{\partial \tilde{H}}{\partial \sigma} \tilde{\Gamma}+\tilde{H} \frac{\partial \tilde{\Gamma}}{\partial \sigma}\right| \leqslant D_{F} \sigma_{F}|\rho| \widetilde{\widetilde{e}} \leqslant J \varepsilon
\end{gathered}
$$

(18) holds because of the Lemma and in view of the fact that $\tilde{H}$ and $\frac{\partial \tilde{H}}{\partial \sigma}$ are bounded functions of $\sigma$. Therefore, $\left|\frac{\partial M(\sigma)}{\partial \sigma}\right| \leqslant \frac{4 J \varepsilon}{d^{2}}=M \leqslant 1$, which holds for $\varepsilon \leqslant \min \left\{\frac{d}{2 \tilde{e}}, \frac{d^{2}}{4 J}\right\}$.

Finally, we have to check that $\sigma_{S}\left(S_{t}, t ; \sigma, c\right)=M(\sigma) \geqslant \sigma_{0}$, where $0<\sigma_{0}<$ $\infty$, as required by the Lemma. Since $\frac{-D_{F} \sigma_{F}}{D_{S}+\rho \tilde{H} \tilde{\Gamma}}=\frac{D_{F} \sigma_{F}}{-D_{S}-\rho \tilde{H} \tilde{\Gamma}} \geqslant \frac{D_{F} \sigma_{F}}{-D_{S}}>0$, we can put $\sigma_{0}=\frac{D_{F} \sigma_{F}}{-D_{S}}$, which completes the proof.

\section{Conclusion}

We extended the analysis of feedback effects of dynamic hedging strategies on the underlying asset in the case of finitely liquid markets to the case of sunk costs of transactions. We showed that the action of program traders leads to an excess volatility of the asset price, the size of which depends on the average size of adjustment and on the average gamma. The former depends on the properties of the adjustment hazard function and on the size of the transaction costs, while the latter indicates how often, on average, a position must be rehedged in order to maintain a delta neutral position. Finally, we showed how 
the fixed point problem for the volatility of the asset price can be solved, to conclude that from a practical viewpoint it may be reasonable to use BlackScholes strategies based on $\sigma_{S}\left(S_{t}, t ; \sigma, c\right)$ for hedging purposes provided that the difference between $\sigma_{S}\left(S_{t}, t ; \sigma, c\right)$ and $\sigma$ is sufficiently small.

\section{References}

[1] Caballero R.J. and Engel E. M. R. A. (1993) Microeconomic Adjustment Hazards and Aggregate Dynamics, Quarterly Journal of Economics, p. 359383.

[2] -.- (1999) Explaining Investment Dynamics in U.L. Manufacturing: A Generalized (S,s) Approach, Econometrica, Vol. 67, no. 4, p. 783-826.

[3] Frey R. and A. Stremme (1997) Market Volatility and Feedback Effects from Dynamic Hedging, Mathematical finance, 351-374.

[4] Grossman S. J. (1988) An Analysis of the Implications for Stock and Futures Price Volatility of Program Trading and Dynamic Hedging Strategies, Journal of Business, vol. 61, no. 3, p. 275.

[5] Henrotte P. (1993) Transaction costs and duplication strategies, Working Paper, Stanford University.

[6] O'Hara M. (1995) Market Microstructure Theory, Blackwell Business.

[7] Platen, E. and M. Schweizer (1994) On Smile and Skewness, Statistics Research Report 027-94 Australian National University Canberra.

[8] Schönbucher P. and P. Willmott (2000) The Feedback Effect of Hedging in Illiquid Markets, SIAM Journal of Applied Mathematics, vol.61, no.1, p. 232-272.

[9] Whalley, A.E. and Wilmott P. (1993) Option pricing with transaction costs, MFG Working Paper, Oxford.

[10] Wilmott P. (2000) Paul Wilmott on Quantitative finance, John Wiley \& Sons, LTD. 